\title{
Medical and Genetic Aspects of Monitoring Women's Reproductive Health in North Ossetia-Alania
}

\author{
Tsallagova L.V. ${ }^{1,2, *}$ Popova L.S. ${ }^{1,2}$ Dzagoeva Z.L. ${ }^{2}$ Gavrilov M.Yu. ${ }^{3}$ \\ Maysuradze L.V. ${ }^{1,2}$ Kabulova I.V. ${ }^{1,2}$ Baroeva M.D. ${ }^{2}$ Gabanov S.T. ${ }^{4}$

\begin{abstract}
${ }^{1}$ Department of pathology of mother and child, Institute of Biomedical Research Branch Vladikavkaz Scientific Center of the Russian Academy of Sciences, Vladikavkaz, Russia. 2 Department of Obstetrics and Gynecology № 1, North Ossetian State Medical Academy of the Ministry of Health of the
Russian Federation, Vladikavkaz, Russia

${ }^{3}$ Pirogov Russian National Research Medical Universityv, Moscow, Russia

${ }^{4}$ North Ossetian State Medical Academy of the Ministry of Health of the Russian Federation, Vladikavkaz, Russia
\end{abstract} \\ *Corresponding author. Email: akusherstvo_1@mail.ru
}

\begin{abstract}
A survey of patients of reproductive age living in environmentally unfavorable and relatively favorable regions was carried out. It has been established that high air and soil pollution with heavy metals have a gonadotoxic effect, primarily violating the central link in the regulation of reproduction. The frequency of reproductive pathology of patients was largely determined by the characteristics of reproductive behavior. Prediction of the pathology of pregnant women and newborns in areas of environmental distress contributed to the identification of preclinical forms of the disease and timely correction of environmentally dependent conditions, which led to a decrease in perinatal morbidity.
\end{abstract}

Keywords: genetic monitoring, pregnancy, heavy metals, reproductive health

\section{INTRODUCTION}

The development of modern medical research in the field of obstetrics and gynecology and medicine as a whole has led to the fact that to date, a lot of information has been accumulated on the mutagenic effect of certain trace elements in areas of adverse effects of environmental factors. The least studied of them are cobalt, cadmium, zinc and lead. In the city of Vladikavkaz, these trace elements are common in soil, in air, and in water in areas of high environmental pollution [1-3]. Given the genotoxicity of the environment, it is relevant to study the genetic effects caused by the effects of these metals on the body of women in North Ossetia-Alania to predict their adverse effects on the health of pregnant women and newborns. Considerable material has been accumulated on the effect of technogenic pollution on the reproductive system [4-7]. It is known that lead belongs to the latter, which is one of the harmful factors of non-ferrous metallurgy enterprises and significantly increases the risk of developing reproductive and perinatal pathologies.

These studies put forward - among the important tasks of medicine - the implementation of measures to improve the quality of genetic monitoring and to prevent the possible adverse effects of environmental factors on the reproductive health of women [8-10].

The aim of our study was a comprehensive study of indicators of medical genetic monitoring of women living in environmental pollution, and improving the effectiveness of preventive and therapeutic measures in preparing them for pregnancy and childbirth.

\section{METHODS AND MATERIALS}

To identify the peculiarities of women's reproductive behavior, special questionnaires were composed from the data of medical documentation of obstetric institutions, information on menstrual and sexual function, the number of spontaneous and medical abortions, the intervals between pregnancies, the use of various methods of contraception and other data that are often not taken into account in statistical documents. 280 retrospective birth histories were studied retrospectively. In the course of a prospective study, general clinical, laboratory (bacteriological, immunogenetic) and functional examination methods were used. 86 women with reproductive dysfunctions with the purpose of pregravid preparation were examined on the basis of the Department of Mother and Child Pathology at the Institute of Biomedical Research of the Vladikavkaz Scientific Center of the RAS. The main group consisted of 56 women who lived in an ecologically unfavorable zone. The control group was represented by 30 residents of relatively ecologically favorable areas. The patients of the main group were divided into two subgroups. In the I subgroup (22 patients), the traditional treatment of threatening preterm delivery and correction of the identified reproductive disorders was carried out. In the second 
subgroup (34 patients), metabolic therapy courses were additionally prescribed, using glycine and limontar preparations, which are antihypoxants and have antimutagenic and detoxifying properties. Moreover, glycine not only protects the cell from the harmful effects of exotoxicants from the outside, but also neutralizes toxins inside the cell. Limontar removes toxic substances and salts of heavy metals from the body, has radioprotective properties. Glycine and limontar have no contraindications, age restrictions and the risk of overdose.

Genetic studies included accounting for chromosomal aberrations (CAs), which was carried out in lymphocytes of human peripheral blood. For analysis of chromosomal aberrations, human peripheral blood lymphocytes were cultured using the macro method according to the generally accepted method. The growth medium consisted of $10 \%$ serum of cattle and $90 \%$ of the Igla medium (Minimum Essential Medium, MEM) with glutamine. The suspension culture of lymphocytes included leukocyte plasma and growth medium in a ratio of 1:9 [1]. Cell division was stimulated with phytohemagglutinin at a dose of $1 \mathrm{~g} / \mathrm{ml}$ of culture medium.

Chromosomal preparations were prepared by applying a cell suspension to clean fat-free and cooled glass slides, dried and stained with $3 \%$ Giemsa stain on phosphate buffer at $\mathrm{pH}=6.8$. At least 100-150 metaphase plates were analyzed for each option.

The results of the studies were statistically processed, the average values of the variational series were determined, the difference method was used with the calculation of the Student's t-distribution and the criteria for the significance of differences in the results of studies (p). The results were visualized using Microsoft Excel spreadsheet.

\section{RESULTS}

A reproductive study of pregnant women's records showed that the age of women ranged from 18 to 46 years and averaged $26.9 \pm 3.2$ years. Despite the fact that the majority of patients were observed in antenatal clinics $(92.4 \%)$, their early appearance at the obstetriciangynecologist accounted for only $48.7 \%$. Menstrual function was realized in most women aged 11 to 15 years $(71.5 \%) ; 5 \%$ of women had menarche before 11 years old, $18.5 \%$ of them after 15 to 16 years. Of the past illnesses, $42 \%$ of women had a history of childhood infections, $38 \%$ were repeatedly exposed to acute respiratory viral infections, influenza, tonsillitis; $29 \%$ had a history of kidney disease; $7 \%$ had diseases of the liver and gastrointestinal tract; $16 \%$ suffered from hypertension and other cardiovascular diseases; $24 \%$ had anemia and blood diseases; $16.7 \%$ noted endocrine diseases: diabetes, obesity, hyperthyroidism and others

The study of obstetric history has shown a high frequency of gynecological morbidity in women of the city of Vladikavkaz. Inflammatory diseases of the female genital organs were noted more often: acute and chronic inflammation of the uterus in $55.7 \%$ of cases, cervical erosion in $41 \%$ of cases, colpitis of various etiologies in $64 \%$ of cases, menstrual dysfunction in $24 \%$ of cases. Menstrual dysfunction manifested more often in the form of oligomenorrhea and hyperpolymenorrhea; $12 \%$ of women had polycystic ovaries, $16.7 \%$ were treated for infertility in marriage from 1 to 7 years; $14 \%$ had uterine fibroids; $8 \%$ were operated on for an ectopic pregnancy and other reasons. The reproductive behavior of women showed a high frequency of medical abortions, large intergenerative intervals, and a low frequency of contraceptive use of a hormonal nature.

A retrospective analysis of the course of pregnancy showed a high incidence of obstetric complications (Table 1).

Early toxicosis occurred in $26 \%$ of pregnant women and was manifested mainly by vomiting of pregnant women of varying severity. There was a high frequency of edema and hypertensive conditions in the second half of pregnancy $(38 \%)$, in $27 \%$ of cases, there was moderate preeclampsia.

These data indicated possible violations in the system of protective mechanisms in patients, violation of microbiota due to a decrease in the barrier function of the lower genital tract. As the studies showed, in patients living in an environmentally hazardous area, a history of spontaneous miscarriages $(29.6 \%)$ and premature birth (35\%) were more often observed, which, apparently, was a result of a decrease in the protective properties of the body.

Table 1 Analysis of pregnancy complications in patients

\begin{tabular}{|l|c|c|}
\hline \multicolumn{1}{|c|}{ Pathology of pregnant } & \multicolumn{2}{c|}{ Total (n=280) } \\
\cline { 2 - 3 } & abs. number & \% \\
\hline $\begin{array}{l}\text { Moderate preeclampsia } \\
\text { Severe preclampsia, } \\
\text { eclampsia }\end{array}$ & 14 & 27.1 \\
\hline Pregnancy anemia & 80 & 3.1 \\
\hline Threatening premature birth & 183 & 31.8 \\
\hline Early pregnancy loss & 46 & 19.6 \\
\hline Postponed pregnancy & 15 & 4.5 \\
\hline $\begin{array}{l}\text { Bleeding in the second half of } \\
\text { pregnancy }\end{array}$ & 18 & 6.2 \\
\hline Amniotic fluid pathology & 24 & 7.1 \\
\hline Multiple pregnancy & 11 & 2.9 \\
\hline Somatic pathology & 217 & 59.3 \\
\hline
\end{tabular}

Changes in the immunoregulatory system of patients were revealed on the basis of immunological examination 
tests in the conditions of industrial pollution by heavy metals, which indicated the depletion of the reserve capabilities of the immune system. When studying the results of interferon status indicators, a significant decrease in the production of a- and y- IFNs was found in most pregnant women exposed to heavy metal salts. Indicators a-IFN averaged 135.0 $\pm 8.2 \mathrm{MU} / \mathrm{ml}$ compared with $234.0 \pm 2.9$ in the control group, y-IFN averaged $43.2 \pm 10.2$ and $109.7 \pm 1.3$ respectively $(\mathrm{p}<0.05)$. These data indicated unfavorable immunological changes in women of reproductive age from areas of environmental pollution, which may be one of the many causes of pregnancy complications and a response of the immunoregulatory and interferon systems to the effects of adverse factors under the extreme conditions.

The frequency of congenital malformations was analyzed by a retrospective method, which made it possible to identify fluctuations during this period in the range of 36.2-49.3 per 1000 newborns, with an upward trend (Table 2).

Table 2 Frequency of congenital

malformations according to the studied records

\begin{tabular}{|c|c|c|c|}
\hline Year & $\begin{array}{c}\text { Number } \\
\text { of births }\end{array}$ & $\begin{array}{c}\text { Number of } \\
\text { newborns with } \\
\text { congenital } \\
\text { malformation }\end{array}$ & $\begin{array}{c}\text { congenital } \\
\text { malformatio } \\
\text { n per 1,000 } \\
\text { births }\end{array}$ \\
\hline 2010 & 8286 & 78 & 36,2 \\
\hline 2011 & 9406 & 82 & 39,3 \\
\hline 2012 & 10303 & 88 & 45,2 \\
\hline 2013 & 10801 & 98 & 48,3 \\
\hline 2014 & 10760 & 87 & 45,1 \\
\hline 2015 & 10261 & 99 & 67,3 \\
\hline 2016 & 10224 & 115 & 63,7 \\
\hline 2017 & 10280 & 108 & \\
\hline
\end{tabular}

Probably, this is due to the fact that in recent years, the necessary reconstruction in ecologically polluted areas of the city to reduce the amount of harmful substances have not been carried out. The period from 2012 to 2017 was characterized by an increase in harmful accumulations in the atmosphere, water bodies and in the soil.

In the process of pregravid preparation in subgroup II, the frequency of the desired pregnancy significantly increased, the pregnancy outcome changed positively with an increase in the number of timely deliveries with a simultaneous decrease in the number of premature births. The treatment and preventive measures scheme with the inclusion of a metabolic therapy complex used in the pregravid period contributed to a decrease in the mutagenic activity of the environment, an improvement in the reproductive health of patients, which contributed to an increase in the overall resistance of the newborn, as well as a decrease in their morbidity.

Evaluation of the protective effect of antihypoxants was carried out according to the criterion of $\mathrm{CA}$ in patients before and after treatment. When carrying out pregravid preparation against the background of the use of broadspectrum metabolites - glycine and limonthar, containing essential amino acids: amino acetic, citric, succinic, and contributing to the removal of toxic heavy metals - the protection coefficient in some cases increased to $65 \%$, the frequency of CA decreased by 2-3 times.

The main indicator of the effectiveness of the therapy in the pregravid period is the onset of a desired pregnancy and its favorable outcome. A study of patients who have become pregnant in the long-term period, one year after the treatment, showed that after treatment significant changes were observed in the subgroup II of pregnant women, where there were almost 4 times less complicated births than in subgroup I $(\mathrm{P}<0.001)$. The features of the birth act in the subgroup I of patients consisted of a large number of complications $(78.5 \%)$ : untimely discharge of waters was noted in $23.5 \%$ of cases, abnormalities of labor, hypertensive conditions, preeclampsia and eclampsia in labor in $19 \%$, bleeding in labor and early postpartum period in $8.5 \%$ of cases. Analysis of the outcomes of pregnancy and childbirth in women of the subgroup I showed that only $52 \%$ of healthy, full-term children were born to them, and women in the II subgroup had $73 \%$ of healthy children. Most often, women of subgroup I gave birth to children with signs of asphyxia of newborns $(48.5 \pm 2.7 \%)$, prematurity $(16.8 \pm 3.2 \%)$, intrauterine infection $(12.3 \pm 7.2 \%)$, in II subgroup the figure were, respectively, $18.0 \pm 2.0 \% ; 4.0 \pm 1.0 \% ; 2.8 \pm 1.3 \%$ $(\mathrm{P}<0.001)$

After treatment, the average weight of newborns in the I subgroup was $3180.0 \pm 670.0 \mathrm{~g}$, that in the II subgroup was $3520.0 \pm 290.0 \mathrm{~g}(\mathrm{P}>0.05)$. A study of the physical development characteristics of children at birth, the main ones being mass, height, head and chest circumference, showed a decrease in adaptive capabilities in the early neonatal period in newborns whose mothers had contact with heavy metals (lead, cadmium, zinc) in the conditions of environmental pollution.

\section{CONCLUSION}

Thus, the reproductive function of women living in ecologically unfavorable areas of the city occurs against the background of high gynecological morbidity, an increased level of chromosomal aberrations and a high frequency of pregnancy pathology. The main areas of prevention of reproductive health disorders, the development of pathology of pregnancy, the fetus and the newborn are the earlier identification of reproductive disorders in patients, cardiovascular pathology, the conduct of differentiated treatment, prophylactic and rehabilitation measures, taking into account the results of medical genetic monitoring. The positive effect of the 
implementation of these medical recommendations indicates the reversibility in the examined revealed at the preclinical stage changes in the reproductive and cardiovascular systems, as well as the adaptive capabilities of the functional system mother-placenta-fetus.

Based on the data presented, it is recommended that medical and genetic monitoring is performed according to the criterion of chromosomal aberrations in peripheral blood lymphocytes regularly, at least 1 time per year, to women of reproductive age exposed to metal pollutants with a high mutagenic effect.

\section{REFERENCES}

[1] L.V. Tsallagova, L.V. Maysuradze, I.V. Kabulova et al., Features of the treatment of bacterial vaginosis in pregnant women living in environmental pollution by metal-pollutants, Med. Bull. of the North Caucasus 3 (2007) 38-41.

[2] I.V. Kabulova, L.V. Tsallagova, L.S. Popova et al., Modern non-drug technologies for the restoration of reproductive function in pregravid preparation of women, Vladikavkaz Med. and Biolog. Bull. 13(20-21) (2011) 62-67.

[3] N.V. Dorshakova, N.I. Gerasimova, L.I. Tarakanova, Man and the environment: a course of lectures, Publ. House of PetrSU, Petrozavodsk, 2003, 273 p.
[4] G.V. Kovalevsky, Essays on immunomorphology, Nauka Sib. Branch, Novosibirsk, 2001, 264 p.

[5] N.A. Chernykh, S.N. Sidorenko, Ecological monitoring of toxicants in the biosphere, Publ. House of RUDN, Moscow, 2003, $430 \mathrm{p}$.

[6] U. Herpin, J. Berlekamp, B. Markert et al., The distribution of heavy metals in a transect of the three states the Netherlands, Germany and Poland, Determined with aid of moss monitoring, Sci. Total Environ. 187(3) (2000) 185-198.

[7] I.M. Trachtenberg, V.S. Kolesnikov, V.P. Lukovenko, Heavy Metals in the External Environment: Modern Hygienic and Toxicological Aspects, Nauka I tehnika, Minsk, 2001, 285 p.

[8] Z.V. Malysheva, I.I. Sokolova, I.F. Tyutyunnik, Ecology and the reproductive system of women, Occupational health 9 (2000) 18-21.

[9] I.S. Savelyeva, Reproductive health and reproductive behavior of modern youth: prospects and ways of optimization, Ph.D. Thesis, Moscow, 2004, 46 p.

[10] O. Loeber, B. Printer, Sexual education, sexual health training needs, Eur. J. of Contracept. \& Reproduct. Health Care 13(2) (2008) 124-131. 